\title{
One-step deposition of hydrophobic coatings on paper for printed-electronics applications
}

\author{
Zehra Gozutok • Omer Kinj · Ilker Torun • Ahmet Turan Ozdemir • \\ Mustafa Serdar Onses
}

Received: 17 November 2018/ Accepted: 15 February 2019/Published online: 25 February 2019

(C) Springer Nature B.V. 2019

\begin{abstract}
The ability to pattern highly conductive features on paper substrates is critically important for applications in radio frequency identification (RFID) tags, displays, sensors, printed electronics, and diagnostics. Ink-jet printing particle-free reactive silver inks is an additive, material efficient and versatile strategy for fabrication of highly conductive patterns; however, the intrinsic wetting properties of cellulose based papers are not suitable to serve as substrates for this process. This study reports one-step and practical
\end{abstract}

Electronic supplementary material The online version of this article (https://doi.org/10.1007/s10570-019-02326-y) contains supplementary material, which is available to authorized users.

Z. Gozutok · I. Torun · M. S. Onses

Nanotechnology Research Center (ERNAM), Erciyes

University, 38039 Kayseri, Turkey

I. Torun · M. S. Onses

Department of Materials Science and Engineering,

Erciyes University, 38039 Kayseri, Turkey

Z. Gozutok

Department of Textile Engineering, Erciyes University,

38039 Kayseri, Turkey

O. Kinj · A. T. Ozdemir ( $\bowtie)$

Department of Electrical and Electronics Engineering,

Erciyes University, 38039 Kayseri, Turkey

e-mail: aturan@erciyes.edu.tr modification of the surface of paper substrates using industrially available materials. The paper substrates were dip-coated with films of hydrocarbon and fluorocarbon based polymeric resins. Ink-jet printing particle-free reactive silver inks on the modified paper substrates followed by fast thermal annealing resulted in highly conductive patterns. The coatings improved the conductivity of the patterns and reduced the number of printing layers required to obtain conductivity. We finally demonstrated fabrication of a printed RFID tag on the coated paper substrates operating at the frequency range of $865-870 \mathrm{MHz}$.

\footnotetext{
M. S. Onses ( $\square)$

UNAM - National Nanotechnology Research Center, Institute of Materials Science and Nanotechnology, Bilkent University, 06800 Ankara, Turkey

e-mail: onses@erciyes.edu.tr
} 


\section{Graphical abstract}

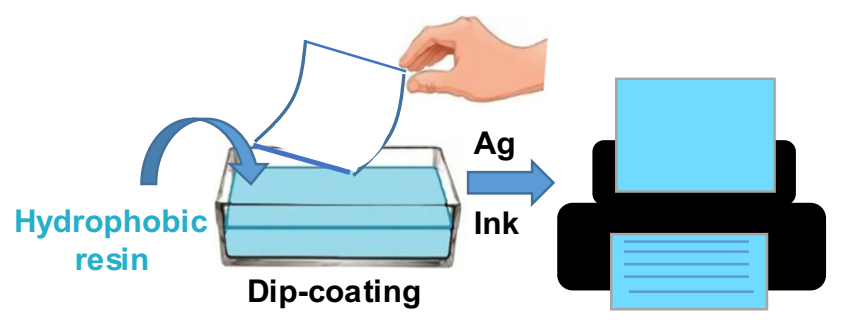

Keywords Printed electronics - Paper - Surface modification $\cdot$ Nanoparticles $\cdot$ Silver

\section{Introduction}

Emerging applications in wearable sensors (Özdemir and Barshan 2014) and devices (Özdemir 2016), biointegrated electronics (Kim et al. 2012), disposable electronics (Lee et al. 2017), wireless electromechanical sensing devices (Zhang et al. 2018) and foldable displays (Nakagaito et al. 2010) require fabrication of electronic devices in unconventional forms. The ability to pattern conductive tracks is one of the most important steps in fabrication of these kinds of electronic devices (Ma et al. 2018). The rigid, planar and thermally stable silicon substrates used in conventional electronics allow for a variety of lithographic processes in conjunction with etching and deposition methods to define conductive features (Xia et al. 1999). These processes are not applicable to flexible, non-planar, and low-thermal budget substrates that are necessary for these emerging applications (Chang et al. 2017; Torvinen et al. 2012). A highly effective approach to fabricate electronic devices on such substrates is one-step printing of conductive materials in an additive manner (Onses et al. 2015; Tekin et al. 2008). Recent developments in preparation of inks based on metallic nanoparticles and carbon nanostructures have presented practical routes for fabrication of printed electronic devices (Kamyshny and Magdassi 2014). A particularly interesting approach developed by Walker and Lewis

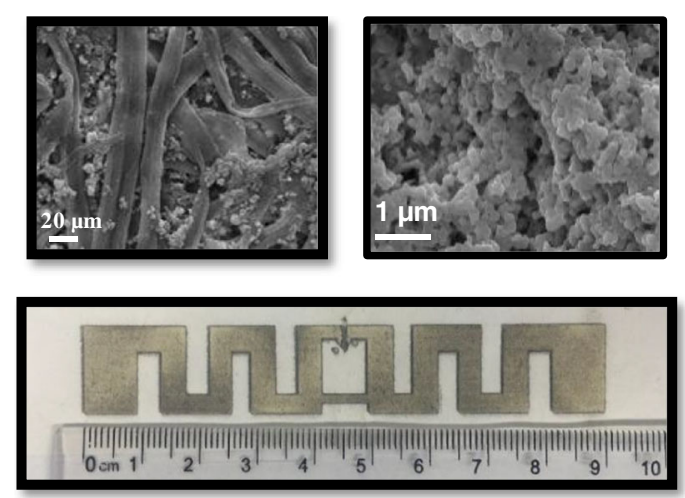

(2012) involved preparation of particle-free aqueous inks that lead to highly conductive patterns upon heating at temperatures as low as $90{ }^{\circ} \mathrm{C}$. The ink is based on a solution of reactive silver precursors and largely overcomes the clogging problems encountered in nanoparticle-based inks. The silver particles form upon printing during evaporation of volatile components. The thermal annealing ensures removal of organic species and results in patterns with conductivities that approach to the bulk conductivity of silver. The broad adaption of this and similar inks in practical applications requires proper engineering of the surface of the low-cost, environmentally benign and flexible substrates.

Paper has attracted considerable interest as a substrate for printed electronics applications, thanks to its low cost, mechanical flexibility, recyclability, and scalable manufacturability (Punpattanakul et al. 2018; Siegel et al. 2010; Tobjörk et al. 2012). As a result, different devices including solar cells ( $\mathrm{Li}$ et al. 2018), touch pads (Mazzeo et al. 2012), batteries (Jabbour et al. 2013), and sensors (Cao et al. 2018) were fabricated on paper substrates. A challenge for printing particle-free aqueous silver and similar inks on paper substrates is its absorbent nature that arises from its structure composed of hydrophilic cellulose fibers. This type of wetting behavior results in spreading of the printed aqueous inks followed by wicking into the substrate (Tobjork and Osterbacka 2011). Besides the enlargement of the printed feature sizes resulting in deterioration of the resolution, the formation of conductive tracks with particle-free inks upon annealing is adversely affected by the intrinsic wetting behavior of the paper substrates (Lessing et al. 
2014). The common approaches to reduce the adsorption of liquids on paper involved laminating with polymers, such as polyethylene (Andersson et al. 2002). Commercial photo papers are good examples to such papers and have been extensively used in printed electronics applications (Allen et al. 2008; Wang et al. 2016). These approaches typically require special infrastructure (e.g. lamination tool) and reduce the flexibility of the substrate. Other strategies to increase impermeability include deposition of hydrophobic particles and plasma processing (Balu et al. 2008; Torun and Onses 2017). These strategies; however, typically bring in additional surface topography and result in extremely repellant surfaces that are not suitable for printing continuous linear features. The solution and vapor-phase deposition of silanes is another effective strategy to improve the wetting properties of paper substrates (Glavan et al. 2014; Tang et al. 2016). The work by Lessing et al. (2014) demonstrated that vapor-phase deposition of silanes made the paper substrate highly suitable for inkjet printing of particle-free reactive inks. The resolution and conductivity of the printed features improved with the increasing hydrophobicity of the paper. This study showed the importance of the wetting properties of the paper for fabrication of conductive features with particle-free aqueous silver inks. Printed electronics applications will greatly benefit from development of solution-processing based complementary strategies for fabrication of hydrophobic paper substrates using versatile, roll-to-roll compatible, low-cost processes and industrially available materials.

In this study, we present a simple and inexpensive approach based on dip-coating of industrially available and ecofriendly resin materials for fabrication of hydrophobic paper to serve as substrates in printed electronics applications. Two types of resins, fluorinated and non-fluorinated, are used in this study. The fluorinated resin is based on alkyl chains of six carbon in length, and therefore conforms to recent regulations on the use of fluorinated compounds. The modification of the surface can be accomplished in durations that are shorter than $5 \mathrm{~min}$ and the method is suitable for a roll-to-roll type processing. Ink-jet printing particlefree reactive silver inks on the modified paper substrates followed by fast thermal annealing results in highly conductive patterns. The conductivity of printed patterns is studied as a function of the number of printing layers on bare and modified paper substrates. We finally demonstrate fabrication of a radio frequency identification (RFID) tag on the modified paper substrates.

\section{Experimental}

Deposition of water-repellant coatings

Office grade paper was purchased from Alkim Inc. and used as received. Two different types of commercially available water repellant resins from Tanatex Chemicals were used in this study. Fluorinated resin, FR, (Baygard ${ }^{\circledR}$ Clean 01) is based on alkyl chains of sixcarbon in length. Non-fluorinated resin, NFR, (Bay$\operatorname{gard}^{\circledR}$ WRS) is a fluorine-free and hydrocarbon based polymeric resin. The deposition of the resins was performed from an acidic aqueous solution. The $\mathrm{pH}$ of the purified water was adjusted to 5.5 by adding acetic acid. The resins were then added to the acidic water solution at a concentration of $100 \mathrm{~g} / \mathrm{L}$. The resins were deposited on the paper substrates by dip-coating. The paper substrates were dipped into the resins for few seconds and then pulled out of the solution at a speed of $20 \mathrm{~mm} / \mathrm{s}$. The substrates were heated at $170{ }^{\circ} \mathrm{C}$ for 2 min after the coating process.

Preparation of particle-free conductive inks and ink-jet printing

Particle-free conductive inks were prepared following the method developed by Walker \& Lewis (Walker and Lewis 2012). To prepare the precursor solution, $1 \mathrm{~g}$ of silver acetate (Sigma-Aldrich) was dissolved in $2.5 \mathrm{~mL}$ of ammonia solution (25\%, Merck) followed by mixing with a stir-bar for tens of seconds at room temperature. Formic acid (95\%, Sigma-Aldrich) with a volume of $0.2 \mathrm{~mL}$ was then slowly added to the precursor solution at a rate of $\sim 4.4 \mu \mathrm{L} / \mathrm{s}$. To settle down and remove the silver particles that formed with the addition of formic acid, the solution was centrifugated at $4000 \mathrm{rpm}$ for $30 \mathrm{~min}$. The clear supernatant was then taken and stored in a fridge $\left(+4{ }^{\circ} \mathrm{C}\right)$ and used as the ink in the printing experiments.

An office-type ink-jet printer (HP Deskjet Ink Advantage 2060) was used for fabrication of conductive patterns on the resin-coated paper substrates. The cartridge was thoroughly cleaned by repeatedly washing in ethanol under sonication and drying with 
air. The particle-free ink was then loaded in the cartridge and printing was performed in the highquality mode (720 dpi) on the paper substrates preheated to a temperature of $170{ }^{\circ} \mathrm{C}$. Upon printing, the paper substrates were annealed at $170{ }^{\circ} \mathrm{C}$ for 2 min.

\section{Structural and wetting characterization}

The morphologies of the paper substrates and conductive tracks were imaged with a Zeiss EVO LS10 scanning electron microscope (SEM) at $25 \mathrm{kV}$. Before SEM imaging, the paper substrates were sputtercoated with a film of gold. The chemical composition of the samples was analyzed using energy-dispersive $\mathrm{X}$-ray (EDX) spectroscopy (Bruker) attached to the SEM and X-ray photoelectron spectroscopy (XPS, Thermo Scientific, K-Alpha). The thin film X-ray diffraction (XRD) analysis was performed using a Rigaku SmartLab diffractometer with $\mathrm{Cu} \mathrm{K} \alpha$ radiation. The static water contact angle of the paper substrates was measured using a contact angle meter (Attension, Theta Lite). The volume of the water droplet was fixed at $4 \mu \mathrm{L}$. At least three measurements were performed over different regions of the substrate.

\section{Electrical resistance of the conductive patterns}

The resistance of the conductive patterns was measured using a two-point probe method. We designed our own probe using four brass alloy spring pins (DESC: spring pins, P/N: ED1420-ND, Manufacturer: Mill-Max Mfg. Corp). This custom designed probe included soft-press springs and enabled accurate measurement of the resistance of the fragile materials. Our probe can precisely follow the material surface, thanks to the flexible spring pins (Supplementary Figure S1). To obtain accurate and reliable results, we used the direct method to determine the resistance using three calibrated precise resistance meter devices (TTI 1906 Digital Multimeter). Our custom design probe has four pins and the first resistance meter was connected between the first and second pins, and the second resistance meter was connected between the second and third pins and the third resistance meter was connected between the third and the fourth pins serially (see Supplementary Figure S1). The distance between the first and fourth pins was $\sim 1 \mathrm{~cm}$ and the distance between the consecutive pins was $\sim 0.33$ $\mathrm{mm}$. The resistance measurements were performed by placing the probe on the paper substrate with the printed conductive pattern with an area of $1 \times 1 \mathrm{~cm}^{2}$.

\section{Fabrication and characterization of the printed} RFID tag

The RFID tag was fabricated using the size and geometry of a commercially available tag (Invengo XCTF-8030A). This ultra-high frequency RFID tag operated at a range of $860-960 \mathrm{MHz}$. The dimensions of the label were $94 \mathrm{~mm} \times 20 \mathrm{~mm} \times 0.21 \mathrm{~mm}$. Received signal strength indicator (RSSI) measurements were obtained via Impinj Speedway reader using an ultra-high frequency near-field antenna (MTI Wireless edge Ltd. MT-249568/NRH) at a frequency range of $865-870 \mathrm{MHz}$. The strength of the received signal was determined using the power level in terms of $\mathrm{dB}$ losses. The RFID chip was cut from the commercial tag and fastened with two plastic clips to the printed tag.

\section{Results and discussion}

Figure 1 presents a schematic description of our approach to fabricate conductive patterns on cellulose based paper substrates. The first step consisted of modifying the surface of the paper with a hydrophobic coating. This step was simply performed by dipping the office-grade paper into a solution of the resin solutions. Two types of industrially available resins were used in this study: one based on hydrocarbon and other based on fluorinated hydrocarbon resin, referred as NFR and FR, respectively. After the coating process, the paper substrates were heated at a temperature of $170{ }^{\circ} \mathrm{C}$ by placing the substrate in between two hot-plates. Particle-free reactive silver ink solution was then ink-jet printed on the coated paper substrates that were preheated to a temperature of $170{ }^{\circ} \mathrm{C}$. A brief annealing at $170{ }^{\circ} \mathrm{C}$ was performed after the printing process for the formation of conductive patterns. This heating step ensured removal of volatile compounds and resulted in reduction of silver ions to metallic silver particles. 


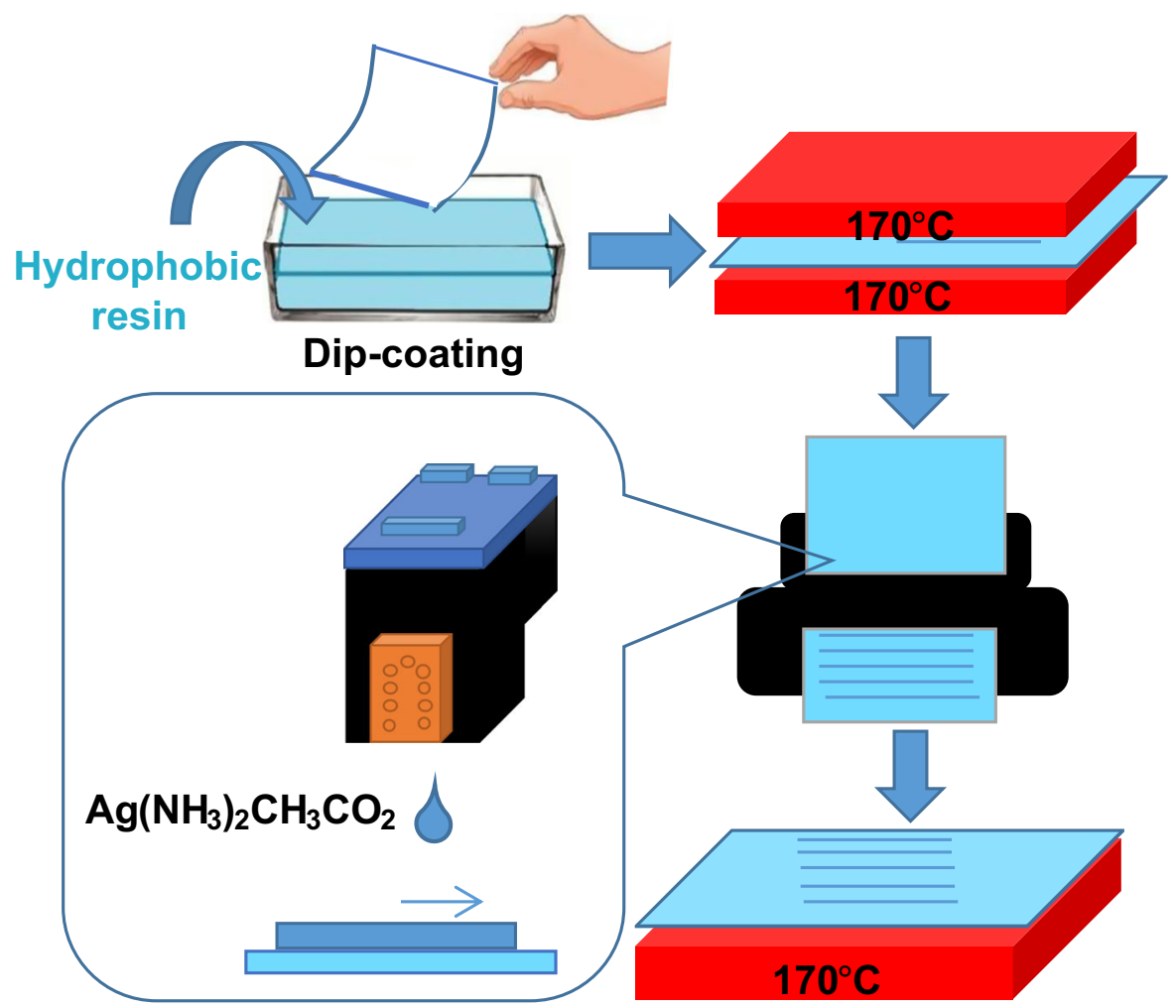

Fig. 1 Schematic description of the process to fabricate conductive patterns on the paper substrate. A hydrophobic polymeric resin was dip-coated on the paper substrate followed by heating in between two hot-plates. Particle-free reactive

Deposition of water-repellant coatings

We first studied the effect of the resin coatings on the wetting and morphology of the paper substrates (Fig. 2). The wetting behavior of the surfaces were characterized via static water contact angle measurements. Initially, the static water contact angle of the bare paper substrate was $105^{\circ} \pm 2^{\circ}$. Both resins increased the hydrophobicity of the paper and the static water contact angles of the NFR and FR coated paper substrates were $136^{\circ} \pm 2^{\circ}$ and $140^{\circ} \pm 2^{\circ}$, respectively. The contact angle of water was higher on the FR coated substrate in comparison to the NFR coated one. This contrast in wettability likely arises from the lower surface energy of fluorinated compounds than hydrocarbons (Kota et al. 2014). SEM images (Fig. 2b) of the substrates showed the presence of the polymeric resins between the cellulose fibers (see Supplementary Figure S2 for a large area image). The hydrophobicity of the FR and NFR coated silver inks were then inkjet printed on the coated paper substrate. A brief annealing following the printing step resulted in the formation of conductive silver patterns on the paper substrate

substrates remained mostly the same with increasing the concentration of the resins and repeating the dipcoating process to deposit multiple layers.

We studied the chemical composition of the paper substrates using XPS and EDX analysis. XPS is a surface sensitive technique, which provides information from the top $5 \mathrm{~nm}$. EDX complements XPS by collecting data from a depth of couple of micrometers and probes both the thin film and substrate. Figure $3 \mathrm{a}$ presents the XPS spectra of the coated paper substrates. Both substrates exhibited characteristic peaks associated with $\mathrm{C}, \mathrm{O}$ and $\mathrm{Ca}$ elements. In the case of the FR coated paper substrate, there was a strong F peak at around $688 \mathrm{eV}$ that relates to the fluorocarbon content of the resin. XPS analysis further showed the presence of $\mathrm{Cl}$ containing species in the FR coated paper. $\mathrm{O}$ and $\mathrm{C}$ peaks of NFR were found at the same peak positions in comparison to the NFR; however, the intensities of these peaks were stronger. On the other hand, a new peak at around 104.6 eV appeared for the 
Non-fluorinated resin (NFR)

(a)

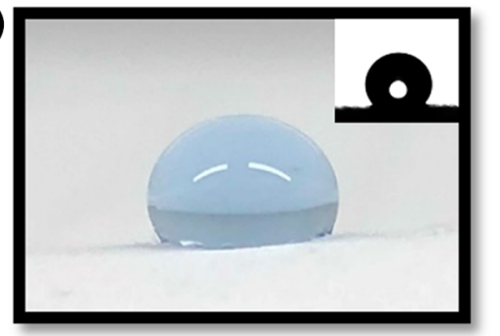

(b)

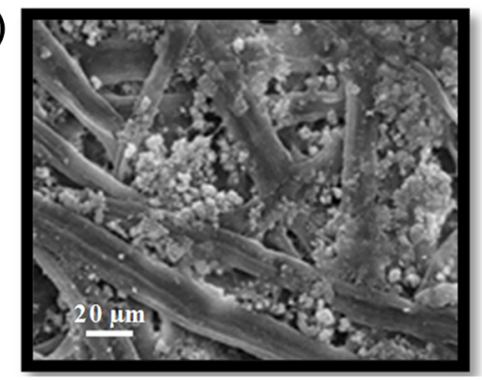

Fluorinated resin (FR)
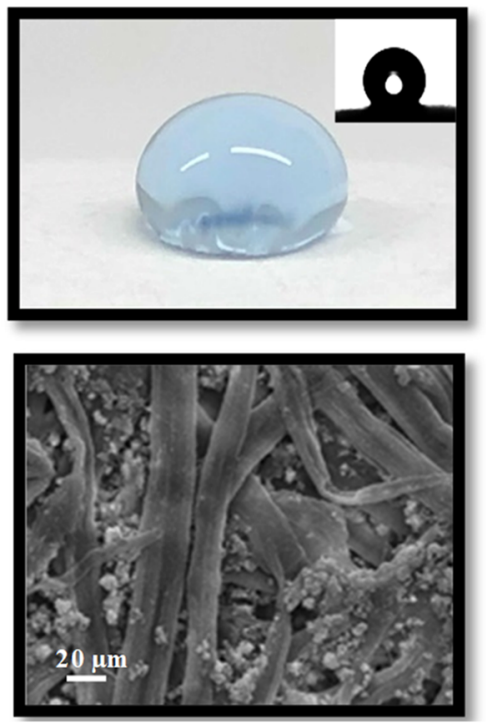

\section{Uncoated}
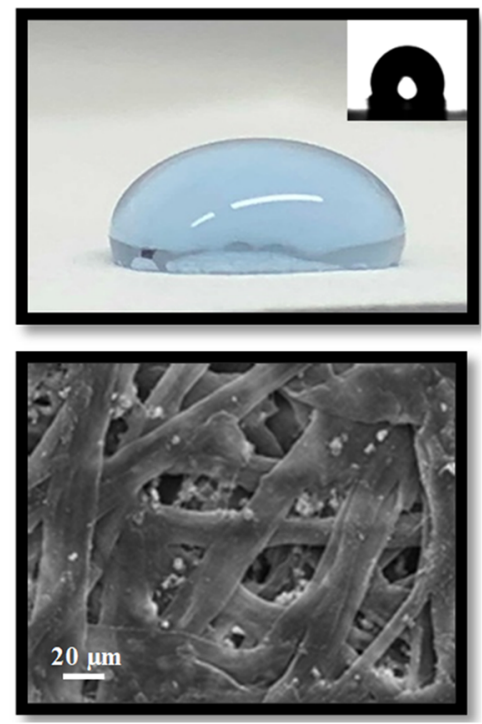

Fig. 2 Wettability and structural characterization. a Pictures of water droplets on the bare and coated paper substrates. The insets present images of the water droplets during the measurement of the contact angle. b SEM images of the substrates

NFR coated sample, which can be assigned to Si2p, suggesting the presence of $\mathrm{Si}$ in the coating layer. EDX analysis (Fig. 3b-d) showed the presence of C, O and $\mathrm{Ca}$ elements in the all substrates. The $\mathrm{Ca}$ peaks were more discernible in the EDX than XPS for the coated substrates, which suggest that the surface of the paper substrates is depleted with this element. These results imply that the uncoated paper substrate contains calcium carbonate species and the coating of substrates with the resins results in the coverage of the substrate with the resins. The relatively hydrophobic nature of the uncoated paper substrate likely relates the calcium carbonate additives present in the paper (Niu et al. 2014). EDX analysis further supports the presence of $\mathrm{F}$ and $\mathrm{Cl}$ in the $\mathrm{FR}$ coated paper and $\mathrm{Si}$ in the NFR coated paper substrates.

Printing of conductive patterns

The resistances of the patterns fabricated by ink-jet printing particle-free silver inks were investigated as a function of the number of printing layers for the coated and uncoated paper substrates. The preheating of the substrates at $170{ }^{\circ} \mathrm{C}$ was important to obtain low resistances over the printed pads. Table 1 lists the resistances of the printed patterns fabricated by passing 5-8 consecutive layers over the same region on the paper substrates. The resistance measurements represent the average values obtained from three different measurements over different regions of the pads. The resistance of the printed pads decreased with the increasing number of layers for the all substrates. The coating of the paper substrates with the resins significantly improved the resistance of the printed patterns. The hydrocarbon-based coating led to slightly lower resistances than the fluorinated one. The resistance of the coated paper substrates was roughly 40-fold lower than the bare paper substrate for the range of layers investigated. We could not obtain reliable resistance measurements on the bare paper substrate up to 6 printed layers, where the resistances of the printed patterns on the coated substrates was as high as $2.64 \Omega$. The lowest resistance was obtained by printing 8 consecutive layers on the NFR coated paper substrates. The significant improvement of the resistances together with the reduction in the number of printed layers required to obtain reliable conductivity showed the promise of the presented coatings.

We further characterized the conductive patterns fabricated by ink-jet printing particle-free reactive silver inks on the coated paper substrates. SEM images presented in Fig. 4a clearly show the presence of high 
(a)

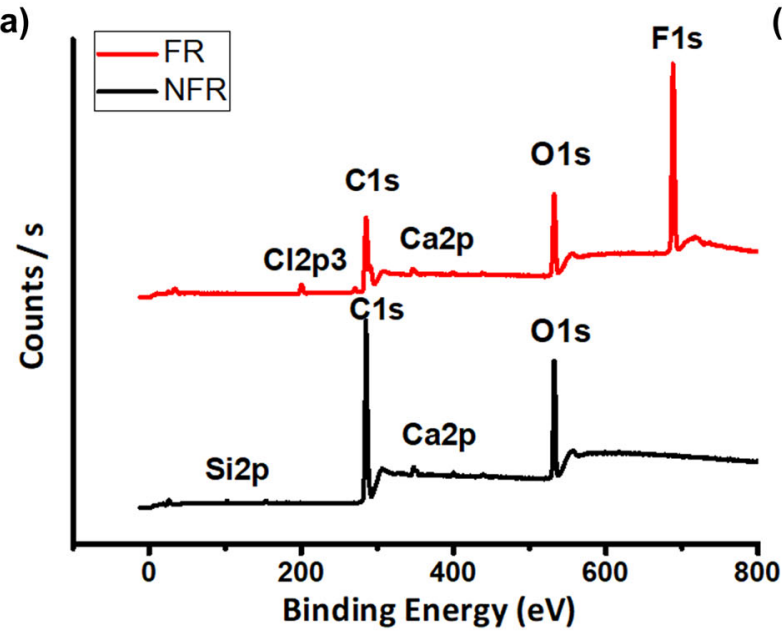

(c)

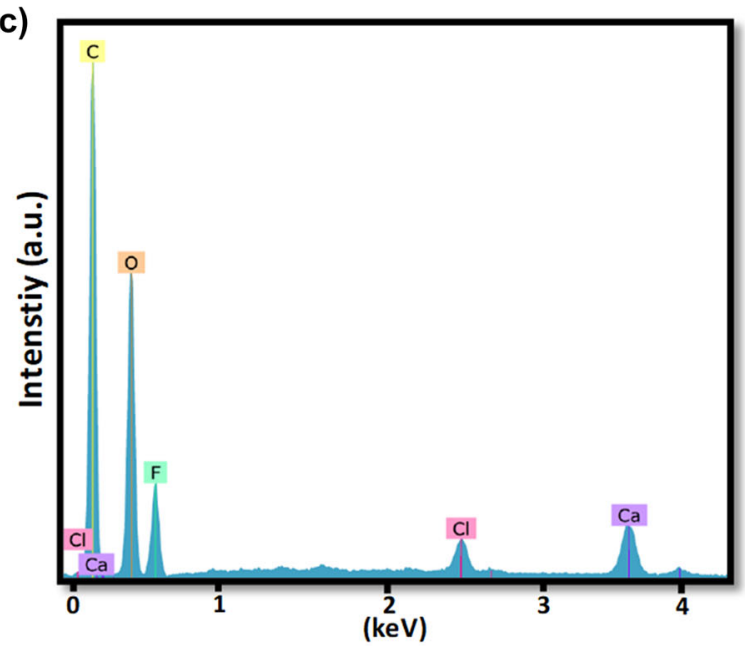

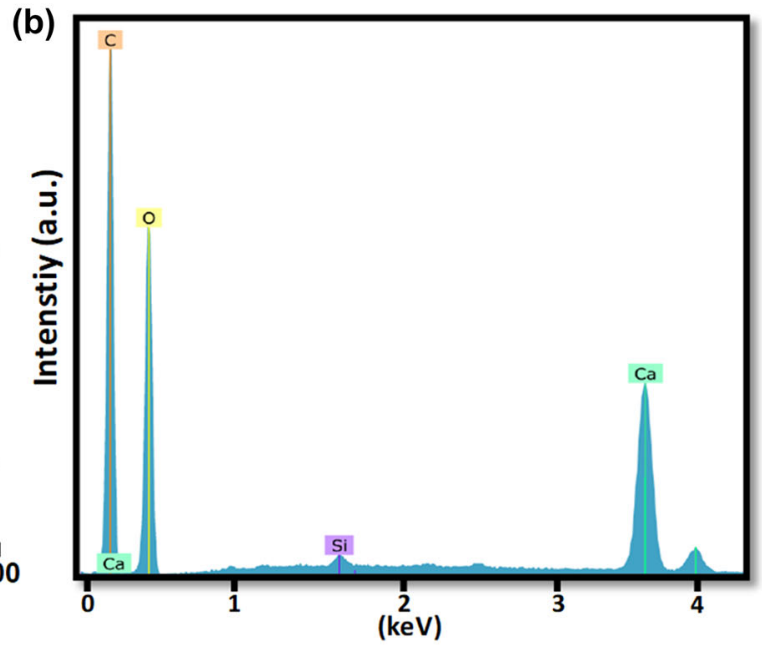

(d)

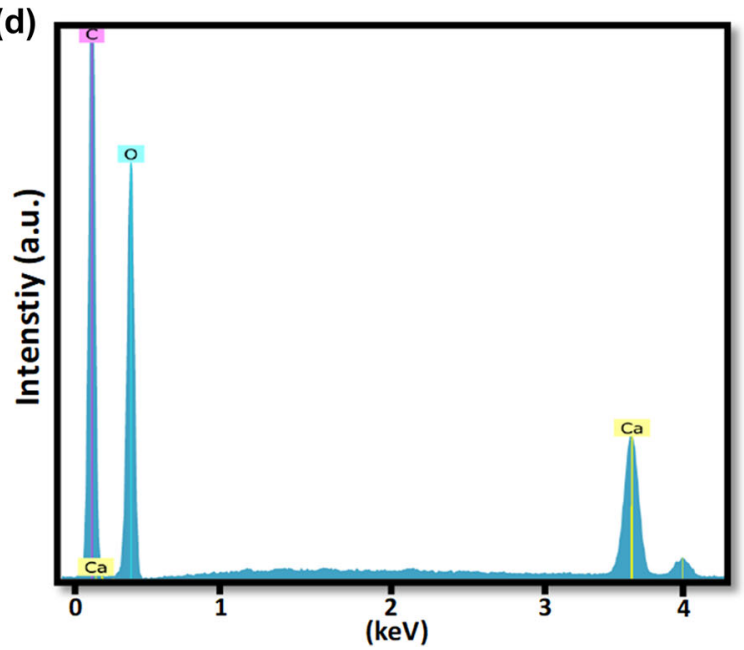

Fig. 3 Chemical composition of the coated paper substrates a XPS spectra of NFR coated and FR coated paper substrates. EDX spectrum for b NFR coated paper, c FR coated paper, $\mathbf{d}$ uncoated paper substrates

Table 1 The resistances $(\Omega)$ of the printed silver patterns on different paper substrates for varying number of printing layers

\begin{tabular}{llcc}
\hline Number of layers & NFR & FR & Uncoated \\
\hline 5 & $2.64 \pm 1.10$ & $15.93 \pm 4.63$ & - \\
6 & $1.42 \pm 0.65$ & $4.66 \pm 3.24$ & $134.5 \pm 40.95$ \\
7 & $0.96 \pm 0.63$ & $1.33 \pm 0.51$ & $37.43 \pm 3.68$ \\
8 & $0.29 \pm 0.03$ & $0.87 \pm 0.03$ & $15.33 \pm 4.83$ \\
\hline
\end{tabular}

The values represent the average \pm standard deviation in the resistance measurements taken from three different regions

density and fine silver particles within the patterns defined by the ink-jet printing followed by the brief thermal annealing. The fibrous structure of the paper was not discernible, since the silver particles merged and formed a continuous layer on the substrate. The silver particles did not exist in the background regions of the coated paper substrate, thanks to the additive nature of ink-jet printing that enables direct delivery of 
(a)

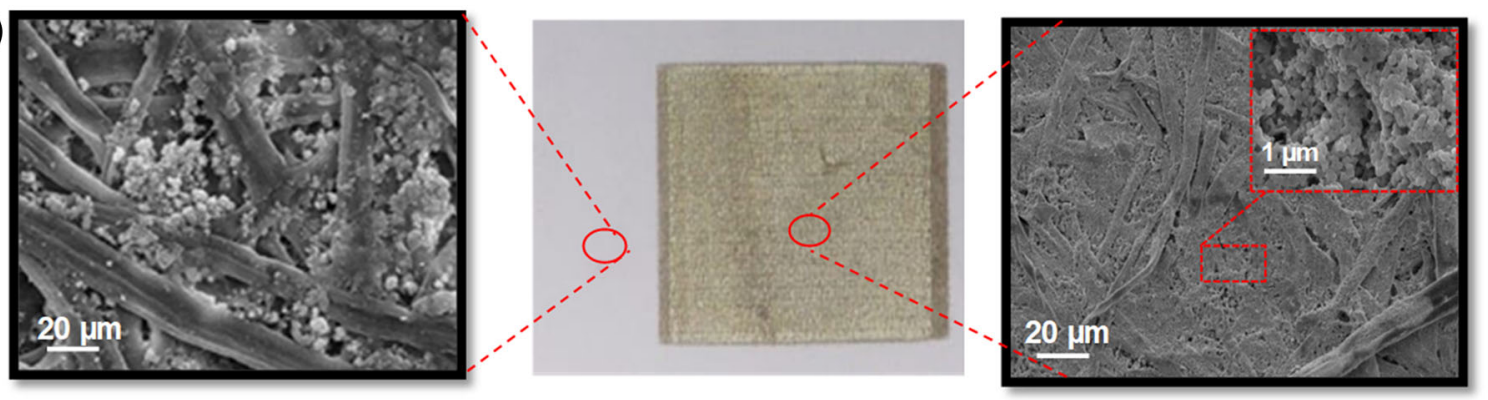

(b)

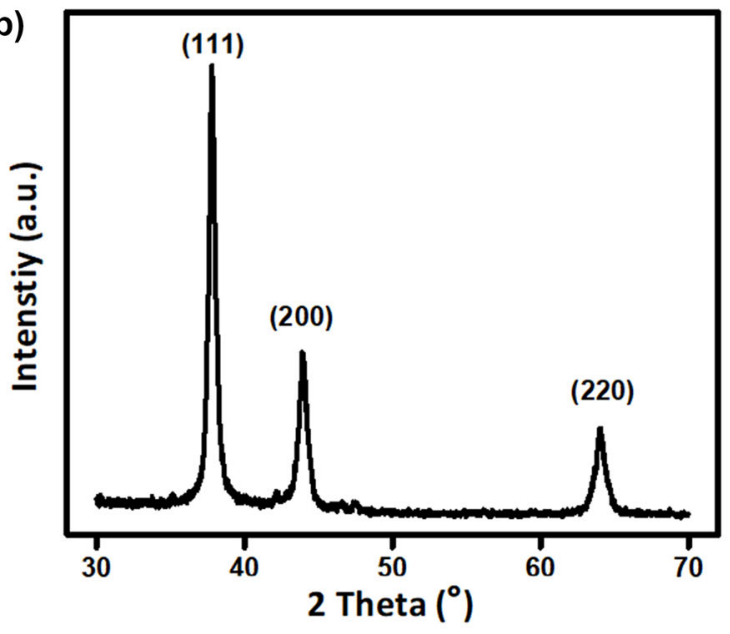

Fig. 4 Structural characterization of conductive patterns fabricated by inkjet printing particle-free reactive silver inks on the NFR coated paper substrates. a SEM images of the paper

materials. The crystal structure and the chemical composition of the conductive patterns were further characterized by XRD and EDX analysis. The XRD pattern of the conductive patterns presented in Fig. $4 \mathrm{~b}$ clearly shows the characteristic peaks that correspond to the (111), (200) and (220) planes of the facecentered-cubic crystal structure (Sakir et al. 2017; Walker and Lewis 2012). The average size of the silver crystallites was $16 \mathrm{~nm}$ as calculated from the Scherrer equation (see Supplementary Material for details). EDX spectrum (Fig. 4c) further confirmed the presence of silver within the printed regions.

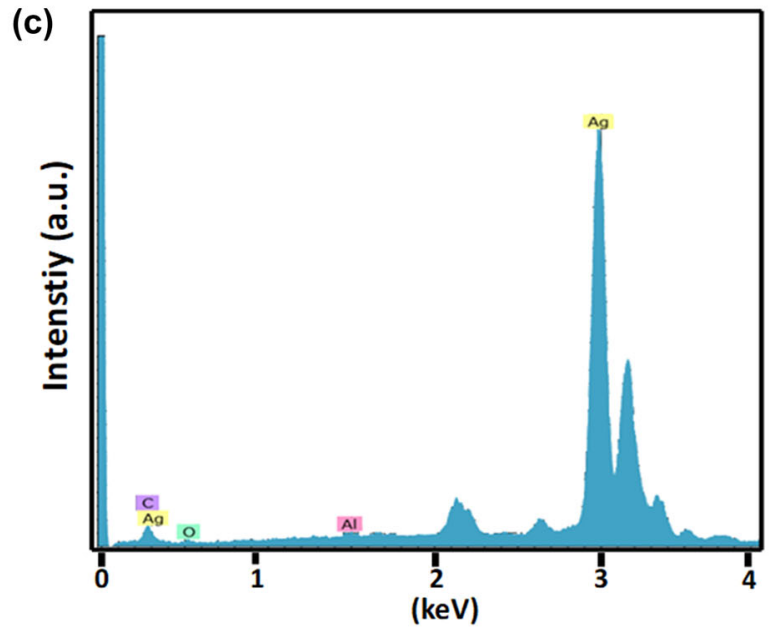

substrates from regions without (left) and with (right) the printed patterns. b XRD pattern, c EDX spectrum of the conductive patterns printed on the NFR coated paper

Fabrication and characterization of the printed RFID tag

We finally demonstrate fabrication of a paper-based RFID tag by inkjet printing particle-free silver inks on the coated paper substrates. For this purpose, we used NFR coated paper substrates and printed 8 consecutive layers of particle-free silver inks. The size of the antenna and frequency of operation impose limitations on maximum attainable gain and bandwidth (Harrington 1960; Wheeler 1975). Compromises have to be made to obtain optimum tag performance and satisfy design requirements. The design (Fig. 5a) of our printed RFID tag followed a commercially available tag to facilitate easy comparison of the performance and use of the off-the-shelf chips. The performances of the printed and commercial RFID tags are compared in 
(a)
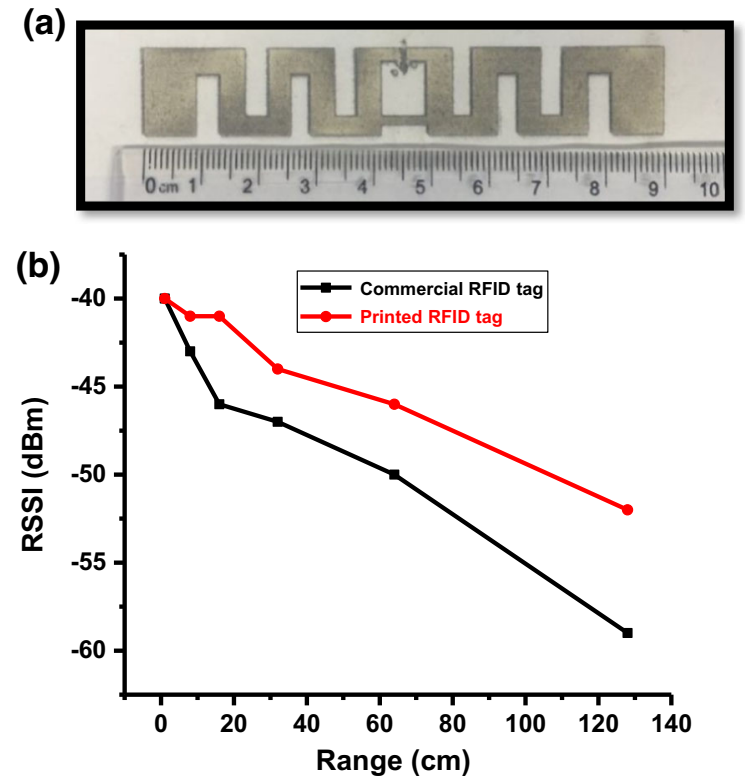

Fig. 5 Printed RFID tags. a Photograph of the RFID tag fabricated by ink-jet printing particle-free silver inks on the NFR coated paper substrate. b The minimum power level to read the tag as a function of the distance for the commercial and printed RFID tags

Fig. $5 \mathrm{~b}$ based on the power losses as the RSSI for varying distances $(1,8,16,32,64,128 \mathrm{~cm})$ between the reader and tag. The performance of the printed tag was better than the commercial tag as indicated by the reduced loss of the power for the entire range of the distances. These results suggest that our printing process is suitable for hardware prototype applications of RF antennas. RF applications are very sensitive for frequency transitions, therefore they are implemented on a printed circuit board, which is time-consuming and costly to fabricate using conventional methods. The ink-jet printing of particle-free inks offers high levels of flexibility in fabrication of conductive patterns overcoming the drawbacks of the conventional methods.

\section{Conclusions}

In conclusion, this study presented versatile, roll-toroll compatible and solution-processing based modification of paper substrates for fabrication of printed electronic devices. The dip-coating of paper substrates with hydrocarbon and fluorocarbon-based resin materials greatly reduced the resistance of the conductive patterns defined by ink-jet printing of particle-free reactive silver inks followed by a brief thermal annealing. In comparison with the bare paper substrates, the conductive patterns could be obtained at much lower number of printed layers, a highly critical parameter for the cost and throughput of the process. The presented approach shows great promise for a variety of applications including sensing, data transmission, wearable electronics and control systems.

Acknowledgments This work was supported by the Research Fund of the Erciyes University (Project No. FBA-2018-8366). MSO acknowledges partial support from the Turkish Academy of Sciences Distinguished Young Scientist Award (TUBAGEBIP). Water repellant resins were kindly supported by SPOT Chemical Industry and Trade Inc.

\section{References}

Allen ML, Aronniemi M, Mattila T, Alastalo A, Ojanperä K, Suhonen M, Seppä H (2008) Electrical sintering of nanoparticle structures. Nanotechnology 19:175201

Andersson P et al (2002) Active matrix displays based on allorganic electrochemical smart pixels printed on paper. Adv Mater 14:1460-1464

Balu B, Breedveld V, Hess DW (2008) Fabrication of "roll-off" and "sticky" superhydrophobic cellulose surfaces via plasma processing. Langmuir 24:4785-4790

Cao R, Zhang X, Tan W, Shen W (2018) Precipitation assay meets low wettability on paper: a simple approach for fabricating patterned paper sensors. Cellulose 25:583-592

Chang J-K, Fang H, Bower CA, Song E, Yu X, Rogers JA (2017) Materials and processing approaches for foundrycompatible transient electronics. Proc Natl Acad Sci 114:E5522-E5529

Glavan AC et al (2014) Omniphobic "R-F paper" produced by silanization of paper with fluoroalkyltrichlorosilanes. Adv Funct Mater 24:60-70

Harrington RF (1960) Effect of antenna size on gain, bandwidth, and efficiency. J Res Natl Bur Stand 64:1-12

Jabbour L, Bongiovanni R, Chaussy D, Gerbaldi C, Beneventi D (2013) Cellulose-based Li-ion batteries: a review. Cellulose 20:1523-1545

Kamyshny A, Magdassi S (2014) Conductive nanomaterials for printed electronics. Small 10:3515-3535

Kim D-H, Lu N, Ghaffari R, Rogers JA (2012) Inorganic semiconductor nanomaterials for flexible and stretchable bio-integrated electronics. NPG Asia Mater 4:e15

Kota AK, Kwon G, Tuteja A (2014) The design and applications of superomniphobic surfaces. NPG Asia Mater 6:e109

Lee S, Seong H, Im SG, Moon H, Yoo S (2017) Organic flash memory on various flexible substrates for foldable and disposable electronics. Nat Commun 8:725

Lessing J, Glavan AC, Walker SB, Keplinger C, Lewis JA, Whitesides GM (2014) Inkjet printing of conductive inks with high lateral resolution on omniphobic "Rf paper" for 
paper-based electronics and MEMS. Adv Mater 26:4677-4682

Li J et al (2018) Conductive regenerated cellulose film as counter electrode for efficient dye-sensitized solar cells. Cellulose 25:5113-5122

Ma Z, Chen P, Cheng W, Yan K, Pan L, Shi Y, Yu G (2018) Highly sensitive, printable nanostructured conductive polymer wireless sensor for food spoilage detection. Nano Lett 18:4570-4575

Mazzeo AD, Kalb WB, Chan L, Killian MG, Bloch JF, Mazzeo BA, Whitesides GM (2012) Paper-based, capacitive touch pads. Adv Mater 24:2850-2856

Nakagaito AN, Nogi M, Yano H (2010) Displays from transparent films of natural nanofibers. MRS Bull 35:214-218

Niu T, Xu J, Huang J (2014) Growth of aragonite phase calcium carbonate on the surface of a titania-modified filter paper. CrystEngComm 16:2424-2431

Onses MS, Sutanto E, Ferreira PM, Alleyne AG, Rogers JA (2015) Mechanisms, capabilities, and applications of highresolution electrohydrodynamic jet printing. Small 11:4237-4266

Özdemir AT (2016) An analysis on sensor locations of the human body for wearable fall detection devices: principles and practice. Sensors 16:1161

Özdemir AT, Barshan B (2014) Detecting falls with wearable sensors using machine learning techniques. Sensors 14:10691-10708

Punpattanakul K, Kraduangdej S, Jiranusornkul N, Chiaranairungroj M, Pimpin A, Palaga T, Srituravanich W (2018) A novel patterning method for three-dimensional paper-based devices by using inkjet-printed water mask. Cellulose 25:2659-2665

Sakir M et al (2017) Fabrication of plasmonically active substrates using engineered silver nanostructures for SERS applications. ACS Appl Mater Int 9:39795-39803

Siegel AC, Phillips ST, Dickey MD, Lu N, Suo Z, Whitesides GM (2010) Foldable printed circuit boards on paper substrates. Adv Funct Mater 20:28-35
Tang Z, Li H, Hess DW, Breedveld V (2016) Effect of chain length on the wetting properties of alkyltrichlorosilane coated cellulose-based paper. Cellulose 23:1401-1413

Tekin E, Smith PJ, Schubert US (2008) Inkjet printing as a deposition and patterning tool for polymers and inorganic particles. Soft Matter 4:703-713

Tobjork D, Osterbacka R (2011) Paper electronics. Adv Mater 23:1935-1961

Tobjörk D et al (2012) IR-sintering of ink-jet printed metalnanoparticles on paper. Thin Solid Films 520:2949-2955

Torun I, Onses MS (2017) Robust superhydrophobicity on paper: protection of spray-coated nanoparticles against mechanical wear by the microstructure of paper. Surf Coat Technol 319:301-308

Torvinen K, Sievänen J, Hjelt T, Hellen E (2012) Smooth and flexible filler-nanocellulose composite structure for printed electronics applications. Cellulose 19:821-829

Walker SB, Lewis JA (2012) Reactive silver inks for patterning high-conductivity features at mild temperatures. J Am Chem Soc 134:1419-1421

Wang F, Mao P, He H (2016) Dispensing of high concentration Ag nano-particles ink for ultra-low resistivity paper-based writing electronics. Sci Rep 6:21398

Wheeler H (1975) Small antennas. IEEE Trans Antennas Propag 23:462-469

Xia YN, Rogers JA, Paul KE, Whitesides GM (1999) Unconventional methods for fabricating and patterning nanostructures. Chem Rev 99:1823-1848

Zhang L, Gupta B, Goudeau B, Mano N, Kuhn A (2018) Wireless electromechanical readout of chemical information. J Am Chem Soc 140:15501-15506

Publisher's Note Springer Nature remains neutral with regard to jurisdictional claims in published maps and institutional affiliations. 